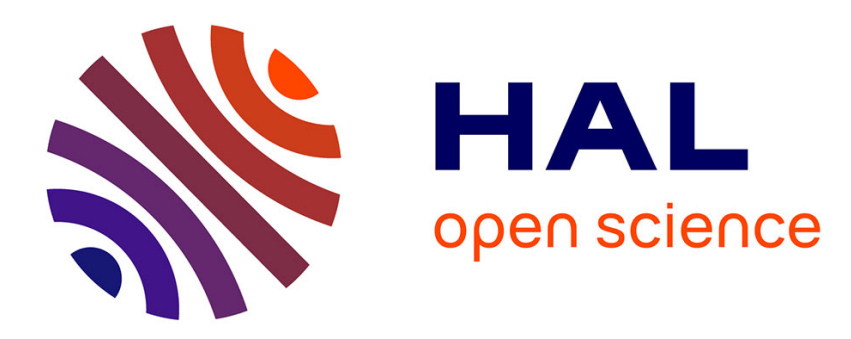

\title{
Penser ensemble les rapports entre individus et communautés à l'époque des Lumières \\ Laurent Loty, Yves Citton
}

\section{To cite this version:}

Laurent Loty, Yves Citton. Penser ensemble les rapports entre individus et communautés à l'époque des Lumières. Dix-Huitième Siècle, 2009, 41, pp.5-26. hal-00848109

\section{HAL Id: hal-00848109 \\ https://hal.science/hal-00848109}

Submitted on 25 Jul 2013

HAL is a multi-disciplinary open access archive for the deposit and dissemination of scientific research documents, whether they are published or not. The documents may come from teaching and research institutions in France or abroad, or from public or private research centers.
L'archive ouverte pluridisciplinaire HAL, est destinée au dépôt et à la diffusion de documents scientifiques de niveau recherche, publiés ou non, émanant des établissements d'enseignement et de recherche français ou étrangers, des laboratoires publics ou privés. 
« Penser ensemble les rapports entre individus et communautés à l'époque des Lumières », avec Laurent Loty, Dix-huitième siècle n ${ }^{\circ} 41$ (2009), p. 5-26

\section{Laurent Loty et Yves Citton}

\section{PENSER ENSEMBLE LES RAPPORTS ENTRE INDIVIDUS ET COMMUNAUTES A L'EPOQUE DES LUMIERES}

\section{AVERTISSEMENT DES EDITEURS}

Ce dossier de Dix-huitième siècle rassemble une quinzaine de d'études participant d'une réflexion collective sur les modes d'existence et de production des individus et des communautés dans les pensées et les sociétés de l'époque des Lumières. Cet ensemble d'articles croise les regards de diverses disciplines (l'histoire des institutions, l'histoire des sciences, l'histoire de l'art, la philosophie, les études littéraires) autour du thème commun de l'articulation du singulier et du collectif. Il fait intervenir des auteurs appartenant à diverses générations (avec une forte représentation de jeunes chercheurs) et en provenance de divers pays - même si l'ensemble est généralement centré sur la France, un quart des contributions propose des incursions dans les domaines germaniques et anglo-saxons.

Quoiqu'il respecte la tradition commune aux numéros passés de Dix-huitième siècle, ce dossier se singularise toutefois en se donnant la forme d'une ébauche de dictionnaire ${ }^{\text {. }}$. Dictionnaire éminemment lacunaire, bien entendu, puisqu'il ne saurait être question de faire le tour (encyclopédique) des questions soulevées par notre thème dans les limites d'une quinzaine d'articles. Dictionnaire volontairement excentrique, par ailleurs, puisque l'un de nos partis pris a consisté à esquiver les problématiques, les auteurs et les textes de référence qui viennent immédiatement à l'esprit lorsqu'on envisage «l'individualisme » des Lumières : on ne trouvera pas d'entrée consacrée à la loi Le Chapelier, au Contrat social, au boulanger égocentré d'Adam Smith, à la statue de Condillac ou à la constitution idéale de Montesquieu. Même s'il reste bien entendu beaucoup à dire sur ces objets canoniques, nous avons invité les contributeurs à revisiter notre thème sous des angles inattendus produisant des effets d'éclairage excentrés : on aura ainsi l'occasion d'envisager des pratiques relevant de la socialité académique, de l'autopromotion d'une ville universitaire ou de la domestication du parterre théâtral, des auteurs comme Marguerite de Lubert, Crébillon fils, Dom Deschamps, Marmontel ou Hölderlin, des genres comme le conte merveilleux ou le drame matrimonial de l'époque révolutionnaire, et des corpus comme le discours physiologiste ou les débats sur l'inoculation. Plutôt qu'une encyclopédie synthétisant - en survol et en surplomb - les grands classiques de «l'individualisme des Lumières », ce dossier propose donc un abécédaire fragmentaire de quelques micro-études qui proposent autant de sondages à travers les multiples couches qui font la complexité, la

\footnotetext{
1 Pour une autre présentation singulière, dont nous croyons qu'elle n'est pas sans rapport avec la diversité de nos enjeux d'ordre épistémologique et politique, au sens large du terme, voyez le numéro spécial de DixHuitième Siècle $\mathrm{n}^{\circ} 5$ intitulé «Problèmes actuels de la recherche » (1973), problèmes posés conjointement, comme le spécifie la page de garde, par Jacques Proust et par la communauté des chercheurs réunis par le numéro. Nous espérons contribuer aussi à répondre à l'appel lancé par Michel Delon dans le numéro qu'il a dirigé sous le titre «Bilan et perspectives de la recherche » (1999), dont la présentation se référait au même numéro 5 de 1973, et analysait avec inquiétude la relative disparition des débats intellectuels, des réflexions épistémologiques ou des entreprises interdisciplinaires, non sans vouloir donner «l'illustration d'une véritable recherche, c'est-à-dire d'un mouvement collectif et inquiet pour déterminer des objets qui nous aident à mieux comprendre le passé et à travers lui le présent. ».
} 
richesse et les nuances toujours locales des nouages esquissés au cours du dix-huitième siècle entre l'individuel et le collectif.

Si la forme du dictionnaire nous a semblé la plus apte à rendre compte du travail réalisé à l'occasion de ce dossier, c'est non seulement parce que l'ordre alphabétique contribue à produire des rapprochements inattendus et suggestifs, traversant les cloisonnements et les chapitrages disciplinaires, tout en laissant toujours ouverte une place intercalaire pour des interventions supplémentaires ${ }^{2}$, mais c'est surtout parce qu'elle offre l'image suggestive d'un mode exemplaire d'articulation $d u$ singulier et du collectif. L'ensemble de ce dossier est en effet le résultat de conversations et d'échanges poursuivis sur trois ans entre les divers participants, depuis la proposition d'un texte programmatique jusqu'aux multiples révisions des textes rédigés, en passant par une riche séance de discussions tenue lors du Congrès de la Société Internationale d'Étude du $18^{\mathrm{e}}$ siècle à Montpellier en juillet 2007. Le jeu de renvois que nous proposons au sein de cet AvantPropos de même qu'à la fin des articles ne signale que quelques-uns des nombreux fils dont s'est tissé le texte commun de cette ébauche de dictionnaire. Comme ce fut notoirement le cas dans l'entreprise coordonnée par d'Alembert et Diderot (quoique bien entendu sans comparaison avec elle), ces fils tirent souvent dans des directions très diverses, qui sont parfois même contradictoires entre elles. L'habit d'Arlequin que revêt immanquablement un dictionnaire issu de la collaboration de plusieurs plumes correspond bien à l'image d'une pensée à la fois commune et individualisante : l'ensemble de ce dossier ainsi que chacune de ses contributions particulières sont effectivement le résultat d'un effort de réflexion éminemment collectif, dont nous espérons qu'il aura permis à chacun des auteurs - et qu'il permettra à chacun des lecteurs - d'affiner sa pensée singulière.

\section{EFFETS DES TEXTES}

Il y a peut-être des textes qui rendent solitaires, et des textes qui rendent solidaires. Que penser de l'Encyclopédie? De la Nouvelle Héloüse? De la Déclaration des droits de l'homme et du citoyen ? D'une revue savante?

\section{INDIVIDUALISME}

Il est sans doute aussi largement admis que fondamentalement correct de considérer l'époque des Lumières comme celle de l'émergence de l'individualisme qui caractérise le mode de civilisation cultivé au cours des derniers siècles par «la modernité occidentale ». Les transformations sociales et idéologiques qui se sont mises en place entre 1650 et 1800 ont sans doute contribué à détacher les hommes des communautés et des traditions qui avaient jusqu'alors défini leur identité à l'aide d'ancrages collectifs qui se sont progressivement érodés, au point de paraître s'être aujourd'hui totalement liquéfiés ${ }^{3}$. La généalogie intellectuelle de ce grand et long bouleversement anthropologique a été retracée par d'importants travaux classiques qui continuent à nourrir les analyses actuelles soucieuses de montrer comment la figure de «l'individu» a émergé du fond des appartenances communautaires pour prendre progressivement une consistance sociologique, politique, juridique, psychologique ou narratologique propre.

En retraçant la naissance de l'économie politique (mise en contraste avec la société de castes indienne), les beaux ouvrages de Louis Dumont ont ainsi conduit à opposer les sociétés hiérarchiques («traditionnelles»), dans lesquelles une structure immuable assignerait à chaque membre du corps social une place et une fonction prédéterminée, aux

\footnotetext{
${ }^{2}$ Voyez Dix-Huitième Siècle, $\mathrm{n}^{\circ} 38$ : «Dictionnaires en Europe », numéro spécial sous la direction de Marie Leca-Tsiomis, 2006.

${ }^{3}$ Voyez les publications récentes de Zygmunt Bauman, Le Présent liquide, Paris, Seuil, 2007 ou La Vie liquide, Rodez, Éditions du Rouergue, 2006.
} 
sociétés égalitaires («modernes », «libérales»), dans lesquelles différents mécanismes juridiques, politiques et économiques reconnaissent à chacun (en principe, sinon en fait) le droit de façonner sa propre trajectoire au sein de l'ensemble social ${ }^{4}$. Dumont prenait en quelque sorte le relai de la distinction classique articulée par Ferdinand Tönnies entre la forme organique (chaude) de la communauté (Gemeinschaft), modelée sur l'unité « naturelle » et étroite de la famille, du sang, de la proximité villageoise ou paroissiale, et la forme chimique (froide) de la société (Gesellschaft) juxtaposant des individus essentiellement séparés, calculateurs de leur intérêt égocentré, se rassemblant ou s'éloignant selon les forces d'attraction ou de répulsion définies par leurs rapports de collaboration, d'alliance et de concurrence ${ }^{5}$.

Entre les deux auteurs, C.-B. MacPherson proposait pour sa part au début des années 1960 un modèle de l'individualisme possessif, selon lequel, à partir de Hobbes et surtout de Locke, l'homme occidental en serait venu à se considérer comme «propriétaire » de son corps, de son énergie, de son temps et de son travail, et comme appelé à intégrer ce type de possession dans la circulation marchande des autres formes de propriétés (mobilières, immobilières, financières) ${ }^{6}$. Dans ses cours de la fin des années 1970, Michel Foucault approfondira cette intuition en décrivant la logique néolibérale comme conduisant chaque individu à se considérer comme l'entrepreneur de soi-même ${ }^{7}$. Dans ses fameux travaux sur la sphère publique et sur l'agir communicationnel, Jürgen Habermas tentait pour sa part de penser l'émergence de nouveaux mécanismes capables de réguler (rationnellement) les interactions de tels individus possesseurs non seulement de leur propriété économique mais aussi de leur initiative politique et de leur pensée propre $^{8}$.

Même si ce thème de l'émergence de l'individualisme, entre le dix-septième et le dixhuitième siècle, s'est parfois fossilisé autour d'oppositions trop abstraites (entre communauté et société, tradition et modernité, État et marché, gouvernement et société civile, égalité et liberté, jacobinisme et libéralisme), il a généré de nombreuses études admirables, dont les plus intéressantes l'exploraient par ses marges plutôt que par ses grands axes (observant par exemple comment les sentiments d'appartenance ou d'amitiés peuvent trouver leur place dans un univers individualiste ${ }^{9}$ ). Au sein de notre dossier, l'article INOCULATION observe ce que les premières explications médicales des contagions et des vaccinations révèlent des nouages perçus alors entre ces deux pôles traditionnels de la problématique individualiste que sont l'intérêt individuel et le bien commun ; l'article

\footnotetext{
${ }^{4}$ Voyez Louis Dumont, Homo Aequalis. Genèse et épanouissement de l'idéologie économique, Paris, Gallimard, 1985 et Essais sur l'individualisme. Une perspective anthropologique sur l'idéologie moderne, Paris, Seuil, 1983.

${ }^{5}$ Voyez Ferdinand Tönnies, Communauté et société. Catégories fondamentales de la sociologie pure (1887), Paris, PUF, 1944.

${ }^{6}$ Voyez Crawford B. Macpherson, La théorie politique de l'individualisme possessif. De Hobbes à Locke (1962), Paris, Gallimard, Folio, 2004

${ }^{7}$ Voyez Michel Foucault, Naissance de la biopolitique, Paris, Seuil, 2004, ainsi que, pour une relecture récente de ce thème, Maurizio Lazzarato, Le gouvernement des inégalités. Critique de l'insécurité néolibérale, Paris, Éditions Amsterdam, 2008.

${ }^{8}$ Jürgen Habermas, L'espace public : archéologie de la publicité comme dimension constitutive de la société bourgeoise (1963), Paris, Payot, 1988.

${ }^{9}$ On peut citer à ce titre - parmi bien d'autres - Pierre Rosanvallon, Le libéralisme économique. Histoire de l'idée de marché (1979), Paris, Seuil, 1989; Gian Mario Cazzaniga et Yves-Charles Zarka (éd.), L'individu dans la pensée moderne, XVI ${ }^{e}$ XVIII ${ }^{e}$ siècles, Pise, Edizioni ETS, 1995 ; Michel Condé, La genèse sociale de l'individualisme romantique, 1986, disponible en ligne sur http://users.skynet.be/conde.michel/Romantable.html ; Jean-Pierre Dupuy, Libéralisme et justice sociale. Le sacrifice et l'envie (1992), Paris, Hachette, 1997, ainsi que Individu et justice sociale : autour de John Rawls, Paris, Seuil, 1988 ; Hélène Merlin, Public et Littérature en France au XVIIe siècle, Paris, Les Belles Lettres, 1994 ; Philip Knee, Penser l'appartenance. Enjeux des Lumières en France, Montréal, Presses de l'Université du Québec, 1995 ; Svein-Eirik Fauskevag, Philosophie de l'amitié, Paris-Oslo, L'Harmattan-Solum Forlag, 2008.
} 
COMMUNION (REPUBLICAINE) propose une reconfiguration originale de cette problématique inspirée par une relecture de textes visionnaires de Hölderlin, dans lesquels la communauté nationale est tendue entre le lien d'amitié et l'horizon d'une Église universelle ; l'article FRATERNITE observe comment les utopies de la période révolutionnaire hésitent à définir ce qui fait des individus (concitoyens ou non) autant de «frères»; l'article SENSIBILITE analyse comment le roman sentimental du dix-huitième siècle situe cette communauté sur le plan «des âmes sensibles », rachetant ainsi par la communion sentimentale la séparation des individus en gestionnaires calculateurs ; quant à l'article ISOLEMENT, il fait entrevoir à travers l'exemple de l'imaginaire marivaudien la figure remarquablement suggestive d'un individualisme dépossessif, dans lequel toute appartenance sociale se voit si radicalement liquidée que le sujet finit par se sentir dépossédé de lui-même, et incapable de profiter des joies promises à la possession. Chacun à sa manière, ces articles font donc d'ores et déjà apparaître, dès cette période d'émergence de l'individualisme, une profonde aspiration à en dépasser la logique auto-mutilante.

\section{INDIVIDUATION}

En même temps qu'elle peut (très légitimement) être lue comme celle du premier triomphe de l'individualisme, l'époque des Lumières peut également apparaître comme celle des premières dénonciations de l'individualisme - voire, plus radicalement, comme celle qui fraie les voies d'un dépassement de l'opposition molaire entre «individu» et « communauté ${ }^{10}$. Lorsque Diderot, dans le Rêve de d'Alembert, s'adresse aux «pauvres philosophes » pour les inviter à «laisser là vos individus » ${ }^{11}$, il déplace par avance le problème des rapports entre individus et communautés du terrain de la sociologie (encore à naître) vers celui de l'ontologie (s'inscrivant dans une tradition théologico-philosophique remontant au moins à Duns Scot, mais marquée plus récemment par les écrits de Spinoza, de Leibniz et de Locke ${ }^{12}$ ) - et il nous invite de ce fait à décentrer notre perspective pour passer des débats sur l'individualisme à une problématique de l'individuation ${ }^{13}$.

$\mathrm{Au}$ lieu d'opposer l'individu à la communauté (tous deux mis au singulier et substantialisés), les problématiques relevant de l'individuation commencent par décomposer les deux pôles que l'individualisme se donnait pour acquis : d'un côté, ce qui se présentait comme un tout insécable (la personne humaine comme individu indivisible) apparaît comme une petite société pluraliste dont la vie dépend d'une suite d'actions et de réactions entre ses parties constituantes ; de l'autre côté, les groupements humains (couples,

\footnotetext{
${ }^{10}$ Voyez Yves Citton, Portrait de l'économiste en physiocrate. Critique littéraire de l'économie politique, Paris, L'Harmattan, 2001 (chapitres XVIII et XIV), ainsi que L'Envers de la liberté. L'invention d'un imaginaire spinoziste dans la France des Lumières, Paris, Éditions Amsterdam, 2006 (chapitres V et VI).

11 Denis Diderot, Le Rêve de d'Alembert (1769), dans Le Neveu de Rameau et autres dialogues philosophiques, Paris, Gallimard, «Folio », 1972, p. 196. Sur la position de Diderot relative à ces questions, voyez les riches articles de Sophie Audidière et al., L'Encyclopédie du Rêve de d'Alembert, Paris, CNRS Éditions, 2006, ainsi que Colas Duflo, Diderot philosophe, Paris, Champion, 2003.

12 Pour de bonnes introductions à cette problématique chez les quatre philosophes évoqués, voyez Gérard Sondag, Introduction à Duns Scot, Le principe d'individuation, Paris, Vrin, 1992, p. 7-84; Alexandre Matheron, Individu et communauté chez Spinoza (1969), Paris, Minuit, 1988; Christiane Frémont, Singularités: individus et relations dans le système de Leibniz, Paris, Vrin, 2003 ; et Etienne Balibar, Introduction et commentaire à Identité et différence. Le chapitre II, XXVII de l'Essay concerning Human Understanding de Locke. L'invention de la conscience, Paris, Seuil, 1998, ainsi que, du même auteur, Spinoza : Il transindividuale, Milan, Edizioni Ghibli, 2002.

${ }^{13}$ C'est bien sur le plan de l'ontologie (et, malgré ses dénégations explicites, sur celui du vieux problème scolastique de l'individuation) que l'Encyclopédie se situe dans l'article «INDIVIDU » en affirmant qu'un sujet « est un individu suivant l'étymologie [dès lors] qu'on ne peut plus le diviser en nouveaux sujets qui ayent une existence réellement indépendante de lui. L'assemblage de ses propriétés est tel que prises ensemble elles ne sauroient convenir qu'à lui. »
} 
familles, villes, communautés, sociétés) apparaissent comme dotées d'une individualité propre (formant certains « corps », dotés d'un certain « esprit » ou d'une certaine « âme »), comparable (jusqu'à un certain point) à l'identité que nous reconnaissons aux personnes humaines. "Quand vous donnerez le nom d'individu à cette partie du tout, c'est par un concept aussi faux que si, dans un oiseau, vous donniez le nom d'individu à l'aile, à une plume de l'aile ${ }^{14}$. Non contentes de voir une composition continue de parties en touts (partiels), depuis la molécule jusqu'à ce «grand individu» que forme l'ensemble de l'univers, les problématiques de l'individuation nous invitent également à rejeter l'idée implicite que l'identité d'un individu puisse jamais être donnée dans la réalité : ce sont seulement les besoins et les limites de nos capacités mentales qui nous conduisent à voir « des individus » (dotés d'un certain isolement dans l'espace et d'une certaine stabilité dans le temps) là où en réalité il n'y a que des processus infinis et pluriels d'individuation. Autant dire qu'un être ne se définit pas par les limites de son corps, mais par les relations qui le constituent ou, pour reprendre encore une fois les formulations du Rêve de d'Alembert, par «la somme d'un certain nombre de tendances » se manifestant par et à travers « une suite d'actions et de réactions ».

$\mathrm{Si}$ certaines pensées de l'individuation ont débouché sur des conclusions très dangereuses en considérant les sociétés humaines comme des touts " organiques », sur le modèle de la ruche, de la fourmilière ou du «corps » animal, assignant ainsi à chacun de leurs «membres » (citoyens, sexes, classes, races) des fonctions spécifiques prétendument « naturelles », toute une tradition de pensée issue du libertinage érudit, de l'épicurisme, du spinozisme et du matérialisme des Lumières inscrit les processus d'individuation dans un univers composé non pas d'essences immuables ou d'équilibres stables, mais d'événements innovateurs et imprévisibles affectant des systèmes fondamentalement «métastables » (c'est-à-dire dynamisés par des tensions internes et par des relations externes qui les poussent nécessairement à sortir de leur état passé et présent). Émergé au siècle de Cyrano de Bergerac et de Spinoza, puis développé par les ténors des Lumières radicales (Meslier, Diderot, Holbach ou Deschamps) - et repris à la fin du vingtième siècle par Gilles Deleuze, Roland Barthes, Toni Negri, Isabelle Stengers, Paolo Virno ou Bernard Stiegler - ce long courant de pensée a sans doute trouvé sa formulation la plus riche et la plus originale dans l'œuvre de Gilbert Simondon (1924-1989), qui représente les processus d'individuation, depuis la formation des cristaux jusqu'à celle du psychisme personnel et des sociétés humaines, comme faisant émerger des modes d'existence individuels à partir d'une réserve de relations «préindividuelles» (constitutives du «milieu ») et toujours au sein de réalités «transindividuelles » (nourries par la participation à un « commun ») en dehors desquelles l'individuation serait impossible ${ }^{15}$.

Ce survol trop rapide de l'histoire et du cadre de réflexion proposé par les pensées de l'individuation permet de cerner l'originalité - et de justifier l'excentricité - des contributions sélectionnées dans ce dossier de Dix-huitième siècle. Ce sont les premiers pas (hésitants et souvent contradictoires) de la réflexion sur l'individuation que retracent quatre de ces contributions. L'article ESSAIM analyse des textes issus du vitalisme montpelliérain se demandant si la « grappe d'abeilles » (chère à Diderot) constitue un « organisme » à part entière ou un simple mode d' « organisation » collective. L'article POLYPE suit pour sa part

\footnotetext{
${ }^{14}$ Diderot, Rêve, p. 196.

${ }^{15}$ L'œuvre de référence de Gilbert Simondon est sa thèse de 1963, enfin publiée dans sa forme intégrale sous le titre L'individuation à la lumière des notions de formes et d'information, Grenoble, Jérôme Millon, 2005. Pour une introduction à la pensée de Simondon, voyez Muriel Combes, Simondon. Individu et collectivité, PUF, 1999, Jean-Hugues Barthélémy, Penser l'individuation. Simondon et la philosophie de la nature, Paris, L'Harmattan, 2005, 2 vol., ainsi que le dossier Politiques de l'individuation : penser avec Simondon, proposé par le $\mathrm{n}^{\circ} 18$ de la revue Multitudes (automne 2004), disponible en ligne sur http://multitudes.samizdat.net/Majeure-Politiques-de-l-.html.
} 
le fil d'échanges épistolaires entre Bonnet et Haller portant sur les implications politiques du type d'identité qu'on attribuera au fameux polype d'eau douce, dont la reproduction par simple scission fait douter s'il faut voir en lui un «individu » (antérieur à la division) ou une «multitude» (en devenir). C'est un problème similaire, quoique relevant d'un genre littéraire totalement différent, que se pose l'article METEMPSYCOSE en étudiant (à la lumière de Locke) des contes merveilleux dans lesquels une «même personne » se voit investie par une multiplicité d' "âmes », parfois rivales entre elles. Et c'est au métaphysicien des Lumières radicales le plus extrémiste en la matière, Léger-Marie Deschamps, qu'est consacré l'article MeEURS (ÉTAT DE), proposant un alignement de l'individuation personnelle sur l'individuation collective (au risque d'abolir toute trace d'individualité).

Quoique sans rapport explicite avec cette tradition de pensée, ce sont encore des questions d'individuation que se pose l'article PUBLIC lorsqu'il analyse, à travers Mercier, La Harpe ou Marmontel, comment la multitude du «parterre » des spectacles théâtraux a été conduite à abandonner ses comportements de foule (traversée de contagions incontrôlables) pour s'individualiser en petits spectateurs disciplinés, embourgeoisés, assis et silencieux. C'est aussi un processus d'individuation (particulièrement velléitaire) que reconstitue l'article UNIVERSITE en observant comment la ville de Göttingen s'est inventé une image de capitale universitaire européenne ainsi qu'une clientèle de d'étudiants issus de la plus haute noblesse. Ce sont encore deux modes très différents d'individuation que met en lumière l'article ESPRIT DE CORPS en comparant les formes de socialité académique pratiquées des deux côtés de la Manche - comme c'est à la recherche d'un mode d'individuation propre au couple et à la famille de l'époque révolutionnaire qu'est consacré l'article DIVORCE.

Bien davantage que des conflits ou que des contradictions entre «l'individu » et «la communauté », ce sont également des modes d'individuation que découvrent les articles Isolement, Reseau, Sensibilite, Solitaires et Utopie (DeS Femmes) au cœur de leurs analyses littéraires : l'anthropologie marivaudienne de l'indigent philosophe illustre une certaine construction parallèle (douloureuse) de l'individu isolé et de la société atomisée ; les chaînes et les réseaux que forment les libertins crébilloniens décrivent précisément un mode d'existence qui circule de projet en projet en ne se supportant dans l'être que par cette circulation même; les âmes sensibles en quête d'une communauté inaccessible s'individuent pour leur part autour de ce manque et de cette aspiration, qui donnent sens aussi bien à leur circulation dans les réseaux de la socialité qu'à leur éventuel refus d'y circuler; Émile et Sophie suit l'élève de Rousseau au moment où son individuation n'est plus assurée ni par un gouverneur surplombant ni au sein d'un couple protecteur, mais s'expose aux aléas d'un monde d'esclavage dont les résistances permettent d'entrouvrir de fragiles zones d'autonomie temporaire ; quant aux conteuses utopistes, c'est justement du sein de l'individuation par couple amoureux qu'elles essaient de tirer l'antidote à l'individuation sérielle régissant la zone de rationalité éternelle fantasmée par certains utopistes masculins.

Toutes ces contributions font apparaître des processus constituants (nécessairement inachevés) au fil desquels les individualités (les sujets, les agents sociaux, les protagonistes romanesques, les narrateurs) et les collectivités (du couple à la famille, au cercle d'amis, à la bande de pirates, à la cité, à la nation et jusqu'à une République européenne des Sciences ${ }^{16}$ ou à une Église universelle) sont amenés à s'individuer ensemble. Chacune de ces études nous aide donc à sentir le fond de relations transindividuelles - le «commun »-

\footnotetext{
${ }^{16}$ Voir sur ce point le $\mathrm{n}^{\circ} 40$ de Dix-huitième siècle dirigé par Irène Passeron, avec la collaboration de Siegfried Bodenmann et René Sigrist, : «La République des Sciences » (2008), où était exploré à travers quelles modalités institutionnelles et quelles formes de subjectivation les érudits et les penseurs de l'Europe des Lumières se sont individués en tant que savants.
} 
nécessaire pour permettre l'émergence de figures individualisées. Deux articles nous donnent également les pôles extrêmes entre lesquels se tendent ces processus constituants : MeEURS (ETAT DE) fournit avec Deschamps le pôle dans lequel le commun anticiperait et résorberait spontanément tout mouvement singularisateur, tandis qu'ISOLEMENT offre l'exemple d'une singularisation rendue impossible par l'absence même de tout commun sinon sous sa forme la plus emblématique qu'est le langage commun, qui, malgré l'indigence des relations sociales, donne toutefois au philosophe-écrivain de quoi s'individuer à travers son énonciation même.

SUPPLEMENTS A LA THEORIE DE L' INDIVIDUATION - On ajoutera quelques suppléments à cette perspective dont la force et les faiblesses se trouvent précisément dans sa puissance unificatrice. Premier supplément: certains usages (indument harmonieux) de l'individuation pourraient négliger l'importance des conflits, et la vertu explicative de visions du monde fondées sur leur prise en compte. Au cœur de la pensée de l'individuation, l'idée que «l'univers dans son ensemble » forme un « seul grand individu » laisse bien supposer que les oppositions locales se résolvent dans une unité ultimement pacifiée. Comment reconnaître à ce grand tout une unité conflictuelle ? Faire (comme Simondon) de la tension le moteur du devenir ne constitue sans doute pas une réponse parfaitement satisfaisante. Second supplément: jusqu'où aller dans l'analogie de fonctionnement entre l'individu (au sens courant du terme) et le collectif, comme entre le naturel et le social ? Réfléchir à partir de l'idée d'individuation exige aussi d'interroger les éventuelles différences de nature entre l'individuation d'un être humain par ce qu'on appelle la conscience et l'individuation d'une communauté par les institutions (ou par cette mémoire qu'est l'écriture de l'histoire). Des articles comme ESSAIM et POLYPE à la fois invitent à opérer le rapprochement entre les processus qui agissent dans ce qu'on appelle la nature et ceux qui sont à l'œuvre dans ce qu'on appelle la société et à s'en défier : car si la société est pensée sur le modèle de la nature, cela peut être que la nature est préalablement pensée sur le modèle de la société - cependant que l'imprévu naturel, comme la régénération du polype, mais aussi l'imprévu social, comme la «régénération » de la Révolution, perturbent heureusement les modèles préconçus. En cela, l'indisciplinarité ne doit pas valoir comme assimilation, mais comme réflexion sur des relations, et comme évaluation du caractère fondé (ou fallacieux) de telle ou telle frontière disciplinaire. Dernier supplément: par comparaison avec la théorie, l'histoire permet de raconter des déroulements et des processus, plutôt que d'en formuler les lois. Ce faisant, plutôt que de viser le fonctionnement général, elle met l'accent sur une histoire toujours singulière, sur le passé spécifique de tel présent précis. Autrement dit : les processus d'individuation relèvent toujours de devenirs concrets, qu'une théorie (forcément abstraite) de l'individuation ne peut que trahir dans leur singularité.

\section{METHODE (INDISCIPLINAIRE ET ACTUALISANTE)}

Ce dossier fait travailler ensemble des «spécialistes » du dix-huitième siècle pour ébaucher un dictionnaire à la fois commun et nourri des singularités de chacun. Il s'inscrit aussi sous un double effort d'indisciplinarité et d'actualisation. Il s'est agi à la fois de mobiliser tout le savoir accumulé par les approches disciplinaires pour se poser des questions qui se situent en deçà, ou au delà, des divisions entre les disciplines et, parallèlement, de mobiliser des connaissances précises des pratiques et des textes du dix-huitième siècle pour nous aider à mieux nous repérer aujourd'hui au sein des problèmes que nous pose notre actualité. 
Le parti pris d'indisciplinarité ${ }^{17}$ repose sur le fait que les nouages de l'individuel et du commun relèvent de problèmes qui doivent être pensés ensemble sur un fond de réalité antérieur aux découpages disciplinaires, ou repensés ensemble contre les effets pervers de la disciplinarisation des savoirs : c'est ainsi que l'étude littéraire des contes merveilleux s'enrichit d'un dialogue avec la philosophie lockienne (voyez METEMPSYCOSE) ou que des analyses relevant de l'histoire de la physiologie débouchent sur la mise à jour d'imaginaires politiques rivaux (voyez ESSAIM et POLYPE). Face au thème qui est le nôtre, il ne suffit toutefois pas de croiser des méthodes déjà constituées au sein des cloisonnements disciplinaires (selon les mots d'ordre traditionnel de pluri-, d'inter-, ou même de transdisciplinarité), mais d'inventer des méthodes inédites qui fassent apparaître l'unité profonde (et profondément in-disciplinaire) des nouages que les savoirs constitués commencent par séparer. La visée de ce dossier consiste donc à ébaucher - parmi bien d'autres travaux - un plan commun de réflexion au sein duquel puissent entrer en communication des objets aussi divers que la production de socialité par les mécanismes des institutions académiques (voyez. ESPRIT DE CORPS) ou par le sentiment amoureux (voyez UTOPIE (DES FEMMES)), la gestion de la cohésion sociale par l'imaginaire matrimonial (dans ses formes juridiques et dramaturgiques, voyez DIVORCE) ou par la lutte contre la variole (voyez INOCULATION). Ce plan d'intégration réflexive nous force à poser la question éminemment indisciplinaire de comprendre ce qu'est « un individu » et ce qui le rattache à « un commun » à travers, grâce à, ou s'il le faut malgré les différentes formes et strates de savoirs (métaphysique, physiologique, psychologique, sociologique, éthique, politique) dont on dispose pour en saisir les manifestations particulières.

Le parti pris d'actualisation ${ }^{18}$ implique que notre connaissance du passé n'exige d'être infiniment affinée que pour nous aider à cerner plus efficacement les problèmes qui se posent dans notre présent. Que la question des articulations complexes régissant la production simultanée et parallèle des individus et de leurs communautés relève d'une actualité quotidienne en notre début de vingt-et-unième siècle n'a guère besoin d'être souligné. Certains contributeurs à ce dossier ont explicitement balisé le jeu de va et vient qui dynamise notre lecture des textes passés à la lumière de nos réflexions contemporaines - par exemple en éclairant Crébillon fils par des notions tirées du Nouvel esprit du capitalisme (voyez RESEAU) ou en informant une analyse d'Émile et Sophie de Rousseau par des suggestions émanant de Marcus Rediker ou de Deleuze et Guattari (voyez SOLITAIRES). D'autres ont tout mis en place pour que les lecteurs soient spontanément amenés à sentir à quel point les discours et les problèmes rencontrés il y a trois siècles résonnent encore dans nos préoccupations contemporaines (voyez UNIVERSITE). Que de telles lectures actualisantes soient ouvertement revendiquées comme telles ou qu'elles constituent le fond discret sur lequel se détache l'analyse, c'est toujours dans la perspective de la pertinence du détour par le dix-huitième siècle pour mieux saisir notre présent qu'a été constitué ce dossier, que cette pertinence tienne aux similitudes entre le passé et le présent, à leurs différences remarquables (et par là éclairantes) ou à l'enquête sur les processus complexes qui mènent de ce passé à notre présent.

\footnotetext{
${ }^{17}$ Voir sur ce point Laurent Loty, «Pour l'indisciplinarité », The Interdisciplinary Century ; Tensions and convergences in 18th-century Art, History and Literature, edited by Julia Douthwaite and Mary Vidal, Oxford, Studies on Voltaire and the Eighteenth Century 2005:04, Voltaire Foundation, 2005, pp. 245-259 ; et «Les savoirs et les mots : effets mystificateurs de la dénomination disciplinaire, de la Renaissance au présent de l'historien », Le Français préclassique (1500-1650), $\mathrm{n}^{\circ} 10$, Actes du colloque international «La dénomination des savoirs en français préclassique (1500-1650) », coordonné par Philippe Selosse avec la collaboration de Volker Mecking et Marthe Paquant, Champion, mars 2007, pp. 21-35.
}

\footnotetext{
${ }^{18}$ Voir sur ce point Yves Citton, Lire, interpréter, actualiser. Pourquoi les études littéraires ?, Paris, Éditions Amsterdam, 2007.
} 


\section{VINGT-ET-UNIEME SIECLE}

Tandis que se répandent dans les médias des appropriations primaires et stéréotypées des «Lumières », réduisant la «Modernité » à une opposition simpliste entre la citoyenneté égalitaire rêvée par Jean-Jacques Rousseau et le marché libre inspiré d'Adam Smith, entre l'étatisme jacobin et le moralisme kantien, il nous semblait important d'inviter des « spécialistes » du $18^{\mathrm{e}}$ siècle à mobiliser leurs connaissances précises de cette époque pour faire voir quelques-unes des nuances, des richesses, des contradictions et des complexités qui en travaillent la pensée et les pratiques institutionnelles. Présenter les Lumières et «la modernité occidentale» comme signifiant «le triomphe de l'individualisme » ou, au contraire, faire remonter au « jacobinisme » l'émergence des étatismes «totalitaires », c'est en effet à la fois s'appuyer sur quelques évidences difficilement contestables et risquer de perpétuer un aveuglement éminemment dommageable. En ne prenant en compte qu'une seule face des racines idéologiques de notre modernité (qu'il s'agisse de sa face d'individualisme menant à une «déliquescence du tissu social » ou de sa face de formation institutionnelle des consciences individuelles), on s'empêche généralement de mesurer les modes particuliers d'agencement $d u$ singulier et $d u$ collectif qu'imaginent et que commencent à mettre en pratique les hommes et les femmes du $18^{\mathrm{e}}$ siècle. Les pensées prétendument «individualistes » des Lumières nous invitent en effet non seulement à valoriser notre individualité, ainsi que la liberté qui est la condition de son développement : du même mouvement, elles nous appellent également à concevoir (et à consolider) un commun qui nous constitue comme sujets humains (à travers des notions comme l'intérêt général, l' «humanité » ou la langue partagée) et à instituer des collectifs (selon des procédures de sélection, d'élection, d'éducation et de formation faisant l'objet de choix intentionnels). Ce dossier espère montrer que nous avons tous intérêt à profiter de la distance que nous donne le détour par le $18^{\mathrm{e}}$ siècle pour mieux comprendre en quoi la production de l'humain (au sein de la nature) consiste en une production de singularités fondée sur une structuration institutionnelle de solidarités multiples, et pour mieux penser ensemble les virtualités d'autres agencements possibles entre l'individuel et le commun dans le $21^{\mathrm{e}}$ siècle qui s'ouvre devant nous. 